\title{
Antiproliferative and apoptotic effects of xanthohumol in cholangiocarcinoma
}

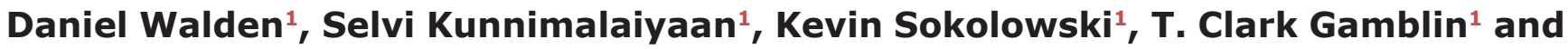 \\ Muthusamy Kunnimalaiyaan ${ }^{1}$ \\ ${ }^{1}$ Division of Surgical Oncology, Department of Surgery, MCW Cancer Center, Translational and Biomedical Research Center, \\ Medical College of Wisconsin, Milwaukee, WI 53226, USA \\ Correspondence to: Muthusamy Kunnimalaiyaan, email: mkunnima@mcw.edu \\ Keywords: cholangiocarcinoma, xanthohumol, Notch 1, apoptosis, cell cycle \\ Received: July 06, $2017 \quad$ Accepted: August 31, $2017 \quad$ Published: September 30, 2017 \\ Copyright: Walden et al. This is an open-access article distributed under the terms of the Creative Commons Attribution License \\ 3.0 (CC BY 3.0), which permits unrestricted use, distribution, and reproduction in any medium, provided the original author and \\ source are credited.
}

\section{ABSTRACT}

Cholangiocarcinoma remains the second most prevalent hepatic neoplasm in the United States with a 5-year survival rate of less than $10 \%$. Currently, no systemic therapy has demonstrated efficacy. Therefore, an urgent need for the identification of molecularly targeted compound(s) remains. The Notch signaling pathway has been shown to be dysregulated in cholangiocarcinoma, exhibiting hyperactivity while also possibly mediating chemotherapeutic resistance. We analyzed the effects of xanthohumol, a prenylated chalcone, on cholangiocarcinoma proliferation utilizing human cholangiocarcinoma cell lines CCLP1, SG-231 and CC-SW-1 while gaining insight into the associated mechanism. Xanthohumol potently reduced cellular proliferation, colony formation, and cell confluency in all three cell lines. Xanthohumol induced cell cycle arrest as well as apoptosis through the reduction of cell cycle regulatory proteins as well as an increase in pro-apoptotic markers (cleaved poly ADP ribose polymerase, cleaved caspase-3) and a decrease in anti-apoptotic markers (X-linked inhibitor of apoptosis and survivin). At the molecular level, xanthohumol reduced Notch 1 and AKT expression in a step-wise and time-dependent fashion, with Notch1 reductions preceding AKT. Additionally, xanthohumol reduced cholangiocarcinoma growth in both CCLP-1 and SG-231 derived mice xenografts. In summary, we show that xanthohumol significantly reduced cholangiocarcinoma growth through the Notch1/AKT signaling axis. Furthermore, known pharmacokinetics and bioavailability of XN supports continued development of treatment for cholangiocarcinoma.

\section{INTRODUCTION}

Cholangiocarcinoma (CCA) is characterized by unregulated proliferation of intrahepatic or extrahepatic epithelial cells lining the bile ducts and accounts for 3\% of gastrointestinal neoplasms [1]. CCA is the second most prevalent hepatic neoplasm with 6,600 new cases of intrahepatic CCA occurring in the United States in 2014, demonstrating an increase of 3.7\% since 2006 [1]. Currently, few therapies exist in the treatment of CCA and the mean survival is 12-months after diagnosis [2]. Poor prognosis is often attributed to the asymptomatic development of the disease, with symptom onset occurring in late-stage CCA, frequently beyond the point of surgical resection [3]. Consequently, the percentage of patients who exhibit a 5-year survival has remained static at 5\% over the past 30 years [4]. There is a potential to develop therapies through understanding intracellular pathways that will contribute towards improving survival of CCA patients.

CCA is frequently associated with a predisposition of biliary inflammatory disease. Primary sclerosing cholangitis, a chronic inflammatory biliary tract disease, is the most frequent predisposing factor for CCA [5]. 
Furthermore, incidence of CCA is significantly higher in Southeast Asia, a region that is endemic to hepatobiliary flukes Opisthorochis viverrini and Clonorichis sinesis [5]. These liver flukes induce potent inflammatory responses which promotes the production of oxidative stress. [6]. The pro-inflammatory state has been linked to potent prosurvival pathways such as PI3K/AKT, Bcl-2 pathway, and Notch signaling, all of which is shown to be overexpressed in CCA [7].

Notch is a highly conserved trans-membrane protein associated with cell fate and development [8]. Upon ligand activation, Notch is cleaved by $\gamma$-secretase, releasing the cytoplasmic domain, which translocates to the nucleus and acts as a transcription factor and regulate various genes expression $[8,9]$. Notch enhances proliferation in many different cancers such as bladder, retinoblastoma, and gastric cancer [10-12]. Studies demonstrated that over-activation of Notch signaling in differentiated hepatocytes led to CCA $[7,13,14]$. Cytoplasmic Notch is overexpressed in CCA and is known to promote migration, positively correlate with tumor size $(>5 \mathrm{~cm})$, and decrease chemotherapeutic sensitivity [15]. Inhibition of Notch induces cell cycle arrest and apoptosis in CCA [16]. We suspect that targeting Notch in CCA may drastically inhibit the oncogenic phenotype, allowing us to better understand and treat CCA in future studies.

Xanthohumol (XN), a prenylated chalcone derived from Humulus lupulus, is a novel compound that has been shown to be efficacious in the prevention of carcinogenesis and neoplastic development [17]. Initial studies using XN on CCA cell lines have shown that a $10 \mu \mathrm{M}$ doses of $\mathrm{XN}$ induces a 5-fold reduction in prostaglandin E2 (PGE2), a product predominantly found to promote CCA migration [17]. We have previously shown that $\mathrm{XN}$ can reduce Notch1 levels in human hepatocellular and pancreatic carcinoma $[18,19]$. However, no studies have shown the anti-carcinogenic efficacy of XN in human CCA cell lines. Here we sought to investigate the in vitro and in vivo effect following $\mathrm{XN}$ treatment in CCA.

\section{RESULTS}

\section{Xanthohumol inhibits proliferation, colony formation, and cell confluency in CCA}

Cellular viability of CCA cell lines was assessed via 3-(4, 5-dimethylthiazol-2-yl)-2, 5-diphenyltetrazolium bromide colorimetric assay (MTT). CCLP-1, SG-231, and CC-SW-1 exhibit concentration and time dependent growth reductions to $\mathrm{XN}$ after 48 and 96 hours (Figure 1A, 1B, 1C respectively). CCLP-1 cells (1A) treated for two days at 5,10, and $15 \mu \mathrm{M}$ concentrations of $\mathrm{XN}$ exhibited a reduced viability of $33 \%, 42 \%$, and $57 \%$, and for four days exhibited reduced viability of $56 \%, 60 \%$, and $84 \%$ respectively compared to control. Statistically significant ( $p$-value $<0.001$ ) values were seen at all three concentrations. SG-231 (1B) had reduced viability after two days of exposure to XN corresponding to $2 \%, 13 \%$, and $31 \%$ and at 4 days $8 \%, 47 \%$, and $80 \%$ at 5,10 , and 15 $\mu \mathrm{M}$ respectively. Statistically significant ( $\mathrm{p}$-value $<0.001$ ) values were obtained at 10 and $15 \mu \mathrm{M}$ concentrations. CC-SW-1 showed $13 \%, 33 \%$, and $59 \%$ at 5, 10, and 15 $\mu \mathrm{M}$ respectively at day two whereas increased reductions were observed at day $4(44 \%, 81 \%$, and $93 \%)$ compared to control (1C). To confirm the anti-proliferative properties of XN, CCA cells were plated to determine their ability to form colonies after XN-treatment. As shown, XN dramatically inhibits the ability of CCLP-1, SG-231, and CC-SW-1 cells to form viable colonies (Figure 1D). Following administration of $\mathrm{XN}$, the ability of CCA cell lines to form colonies is greatly reduced even at the lower concentrations $(5 \mu \mathrm{M})$ tested. Administration of $\mathrm{XN}$ resulted in a dose and time-dependent inhibition of cellular proliferation as well as colony formation. Increased concentrations of $\mathrm{XN}$ reduced the confluency of all three cell lines in a time and dose-dependent manner as measured by the cell confluence real-time kinetics (Figure 1E).

\section{Xanthohumol induces cell cycle arrest through reduction of cyclin $D$ and its binding partners CDK}

To determine the etiology of the growth suppressive nature of $\mathrm{XN}$, we analyzed the quantity of cell cycle markers in three CCA cell lines. CCLP-1 cells showed reductions in cyclin D3, cyclin D1, cyclinE1, and CDK4 suggesting that $\mathrm{XN}$ treatment prevents cell cycle progression through the G1 phase (Figure 2A). SG-231 displayed $\mathrm{XN}$ induced reductions in cyclin $\mathrm{D} 3$, cyclin $\mathrm{E} 1$, as well as in CDK4 (Figure 2A). In CC-SW-1 cells, XN treatment reduced the levels of cyclin D1, cyclin D3, and CDK4; suggesting that $\mathrm{XN}$ causes cell cycle arrest at the G0/G1 phase of the cell cycle (Figure 2A). Additionally, all three cell lines elicited an increase in p21 expression. These findings suggest the observed growth restriction and subsequent cell death is caused by cell cycle arrest and the induction of apoptosis.

\section{Xanthohumol induces apoptosis in CCA cells}

When there is a delay in DNA repair during the cell cycle check point, cells undergo apoptosis. Western analysis revealed that all three cell lines expressed an increase in cleaved poly ADP ribose polymerase (PARP) and a reduction in the pro-survival protein, survivin indicating apoptosis (Figure 2B). Additionally, all three cell lines showed reductions in X-linked inhibitor of apoptosis protein (XIAP) expression in response to XNtreatment. CCLP-1 showed a significant reduction in XIAP following $5 \mu \mathrm{M} \mathrm{XN}$ concentrations, while SG- 
231 and CC-SW-1 exhibited a gradual, dose-dependent suppression. These apoptotic results were confirmed by luminescence assay which measures caspase-3 and -7 activities. As shown in Figure 2C, there was a significant increase in luminescence with increasing concentrations of XN treatment. 15uM concentrations of XN caused 9, 5, and 5-fold increased luminescence for CCLP-1, SG-231, and CC-SW-1 respectively. Taken together, these results suggest $\mathrm{XN}$ reduces cell proliferation via cell cycle arrest and eventual apoptosis.

\section{Xanthohumol induced alterations in Notch1/ AKT signaling pathways}

Previously we have shown that $\mathrm{XN}$ reduces Notch1 in pancreas and hepatocellular cancer $[18,19]$. To evaluate
A)

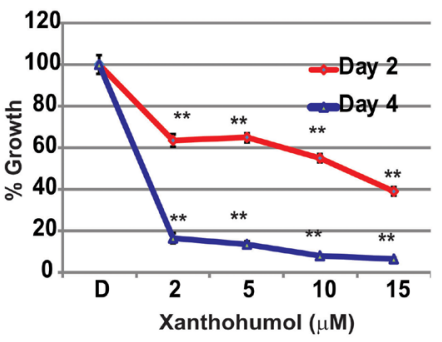

B)

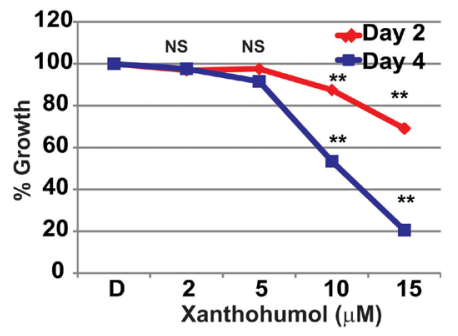

C)

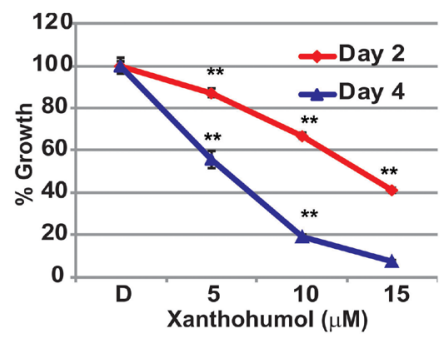

D)

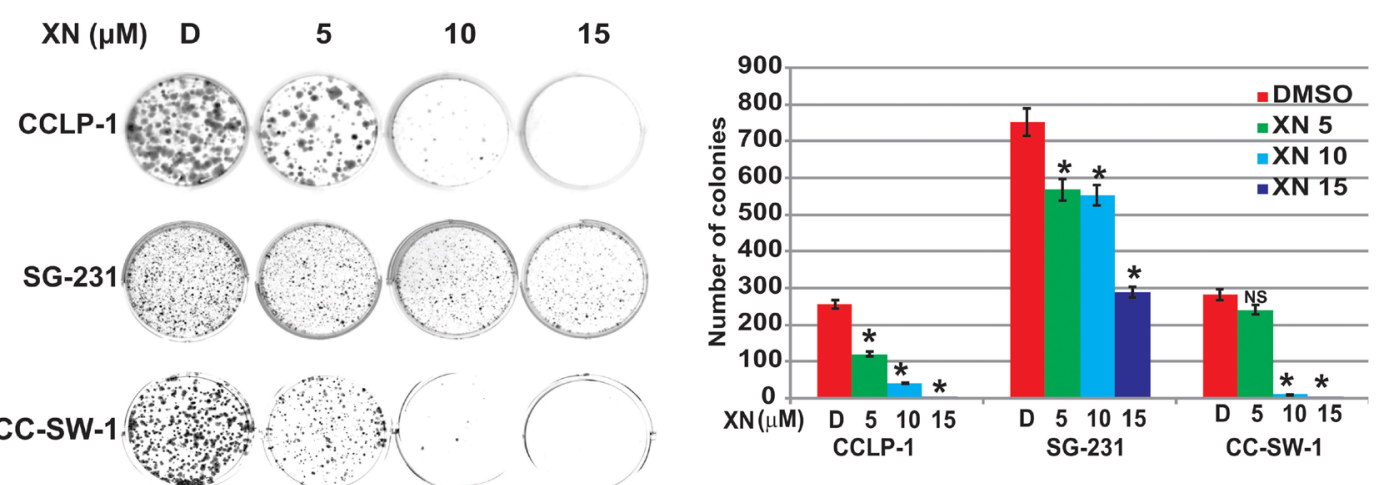

E)
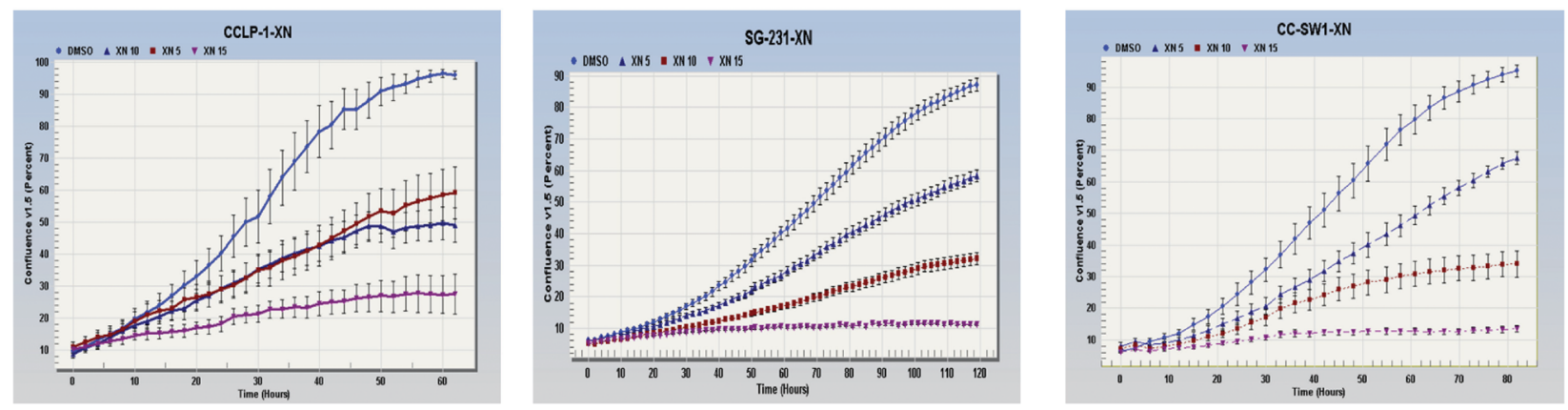

Figure 1: Effects of XN on CCLP-1, SG-231, and CC-SW-1 cellular proliferation. (A) Cells were treated with indicated concentrations of XN and cell proliferation was measured by MTT assay. Statistically significant $(\mathrm{p}<0.001)\left({ }^{* *}\right)$ growth suppression was observed in CCLP-1 (A), SG-231 (B), and CC-SW-1 (C) cells when treated with XN in both 2 and 4-day treatments (NS, not significant). (D) XN treatment inhibits the clonogenic ability of CCA cell lines in colony formation assay. Bar graph shows the number of colonies formed after XN treatment (E) Cells were treated with indicated concentrations and cell proliferation was monitored in real time with the continuous presence of XN. The cells were photographed and the cell confluence was calculated using IncuCyte 2011A software. The changes in cell confluence was used as surrogate marker of cellular proliferation. Significant growth suppression was observed with increasing concentrations of XN. These were statistically significant. 
A)

CCLP-1

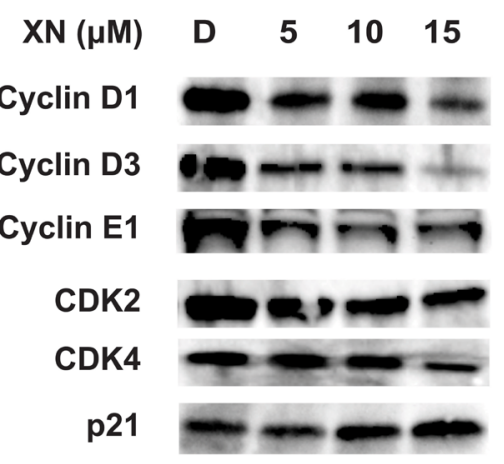

B)

\section{PARP \\ Cleaved PARP}

XIAP

Survivin

GAPDH

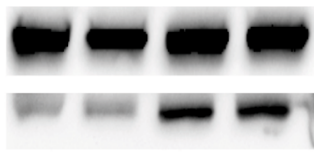

$m$
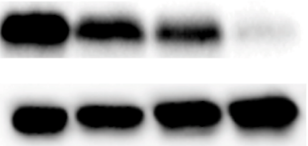

SG-231

$\begin{array}{llll}\text { D } & 5 & 10 & 15\end{array}$
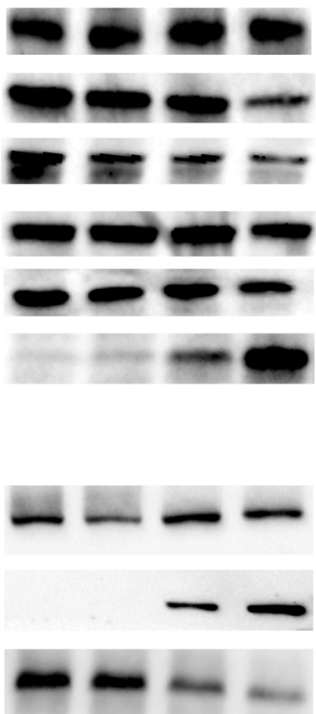

$0=$

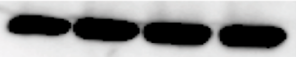

CC-SW-1
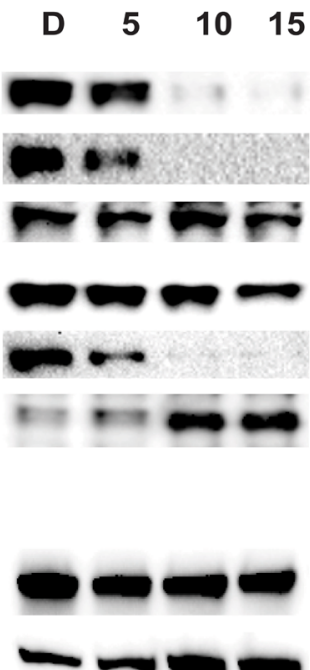

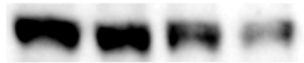

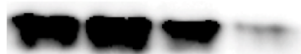

$40 \mathrm{CO}$

C)

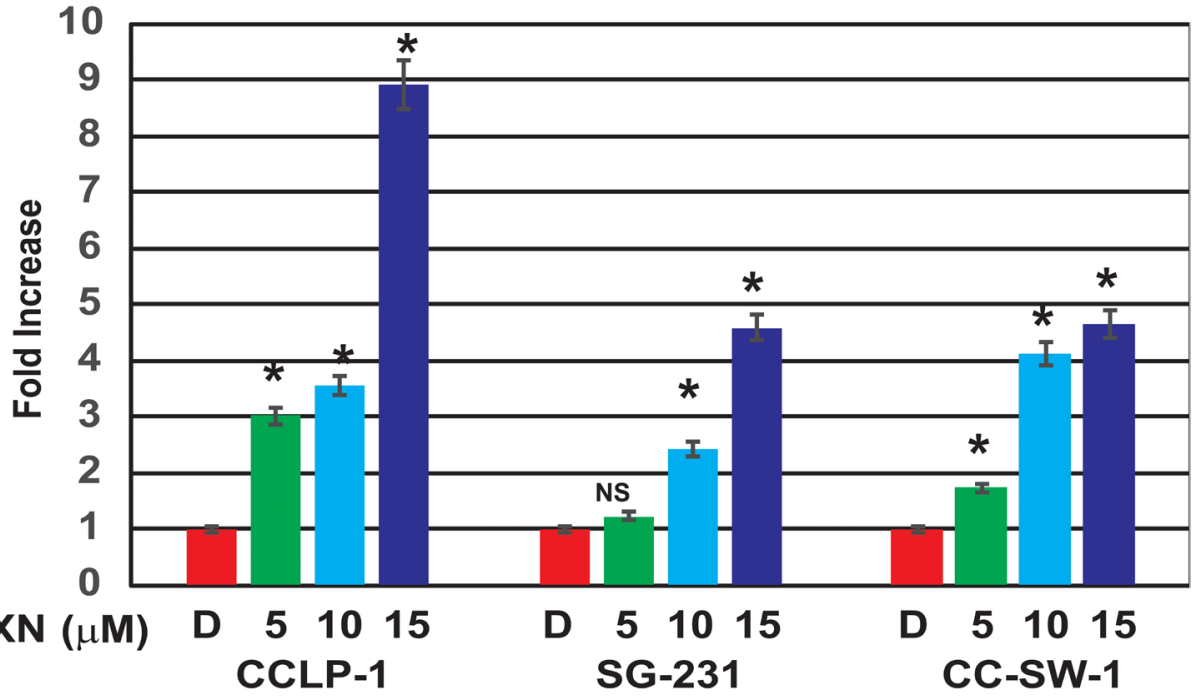

Figure 2: XN induces cell cycle arrest and apoptosis in CCA cell lines. (A) Treatment with XN increases p21, cyclin-dependent kinase inhibitor, in all three cell lines. Key cell cycle regulators cyclin D1, Cyclin D3, Cyclin E1, and CDK2 were reduced in CC-SW-1 whereas cyclin D3 and cyclin E1 and CDK2 and CDK4 were reduced at various levels in CCLP-1 and SG-231. (B) XN-treatment induces apoptosis as evidenced by the increase in cleaved PARP in three cell lines. Furthermore, pro survival protein survivin as well as antiapoptotic protein XIAP was reduced with XN-treatment. Glyceraldehyde 3-phosphate dehydrogenase (GAPDH) is shown as a loading control. (C) Caspase- 3 and -7 activities were measured by caspase Glo3/7 assay $\left({ }^{*}, \mathrm{p}<0.05\right.$ compared to control in three cell lines tested; NS, not significant). 
the mechanism of reduced cellular viability of XN-treated CCA cells, Western blot analysis of cell regulatory signaling pathways was performed. Western blot analysis revealed CCLP-1, SG-231, and CC-SW-1 exhibited dosedependent reductions in Notch1 compared to control treatment (Figure 3A). Furthermore, Notch reduction was associated with reduction in phosphorylation of AKT, a key downstream mediator of the PI3-K/AKT pathway (Figure 3A).

\section{Reduction in Notch1 expression preceded phosphorylated AKT reduction}

Determining whether reduction in Notch1 preceded, occurred concurrently, or because of decreased AKT phosphorylation is critical to evaluate. We carried out western analysis of SG-231 cells treated with $\mathrm{XN}$ at various time points to delineate this issue. As shown in Figure 3B, reduction in Notch1 was observed as early as $12 \mathrm{hrs}$ after XN treatment. At 24hrs the Notch1 was further reduced. There was no reduction of phosphorylated AKT at $12 \mathrm{hrs}$ or even at $24 \mathrm{hrs}$ after treatment suggesting that Notch1 reduction precedes AKT phosphorylation.

\section{Xanthohumol treatment reduces tumor growth in mouse xenograft model}

To examine the effect of $\mathrm{XN}$ treatment in vivo, we performed a xenograft study using CCLP-1 and SG231 cell lines in an immunodeficient, athymic mouse model. Cells were injected subcutaneously into the flank of athymic nude mice. The mice were intraperitoneally injected with $\mathrm{XN}$ at $125 \mu \mathrm{g}$ per mouse $(5 \mathrm{mg} / \mathrm{kg}$ body wt of approximately $25 \mathrm{~g}$ mouse) every other day for 16 days. In both cell lines, the XN treatment reduced tumor growth significantly (Figure 4A and 4B). In mice injected with SG-231 cells, tumor burden was substantially restricted. 8 days after injection untreated mice showed a $427 \%$-fold increase in tumor size compared to a $153 \%$ change in XN treated mice. After 16 days' control and $\mathrm{XN}$ treated mice demonstrated $1308 \%$ and $255 \%$-fold changes, respectively. Additionally, CCLP-1 injected mice

A)

\section{CCLP1}

SG-231

CC-SW-1
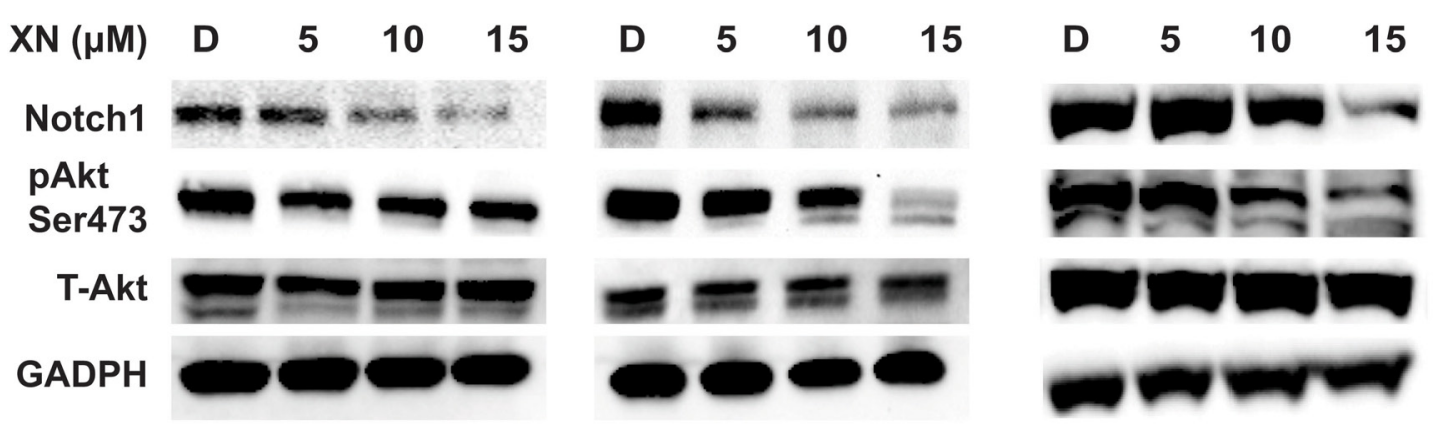

B)

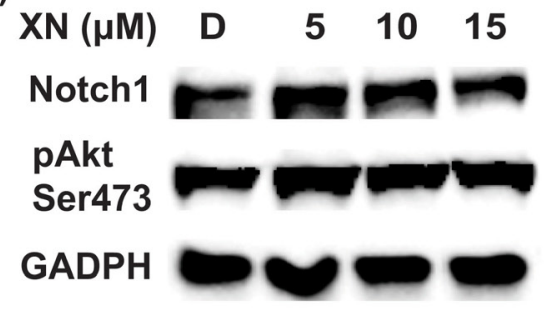

$6 \mathrm{hr}$

SG-231

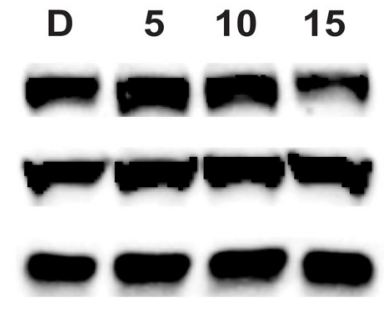

$12 \mathrm{hr}$

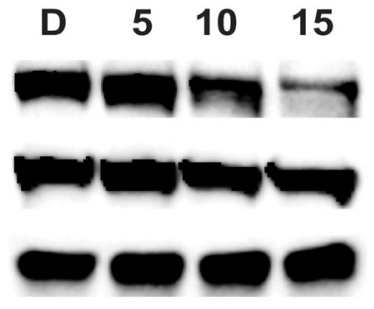

$24 \mathrm{hr}$

Figure 3: XN treatment alters Notch1 and PI3-K/AKT pathway. (A) Dose-dependent reductions in Notch 1 in CCLP-1, SG231, and CC-SW-1 correspond to increasing dose of XN were observed after 72 hours of XN treatment. Phosphorylated AKT at serine 473 (p-Akt Ser 473) is reduced when compared to total cellular AKT. Glyceraldehyde 3-phosphate dehydrogenase (GAPDH) is shown as a loading control. (B) Time course experiments in SG-231 showed Notch1 reduction at $12 \mathrm{hr}$ after XN treatment whereas no reduction in phosphorylated AKT at ser473 position even after $24 \mathrm{hr}$ treatment. Glyceraldehyde 3-phosphate dehydrogenase (GAPDH) is shown as a loading control. 
demonstrated tumor shrinkage as a result of XN treatment. After 8 days of treatment control mice demonstrated 381\% change in tumor volume compared to a $\mathrm{XN}$ treated mice which showed a $13 \%$ decrease in tumor volume size. After 16 days, control mice had a tumor volume $853 \%$ larger than on day 0 , compared to XN treated mice, which had a $78 \%$ reduction in tumor volume. Here we show that, in vivo, $\mathrm{XN}$ not only has the capacity to reduce the growth of CCA, but also the ability to actively decrease tumor burden.

\section{DISCUSSION}

Cholangiocarcinoma is a disease characterized by limited symptomatology and aggressive metastasis leading to significantly high mortality. Consequently, there is a low possibility of surgical intervention [3]. Surgical resection remains the only curative treatment for CCA, yet few are candidates due to late stage presentation. Surgical candidates unfortunately have a poor prognosis, as they are typically limited to a 12-15-month median survival
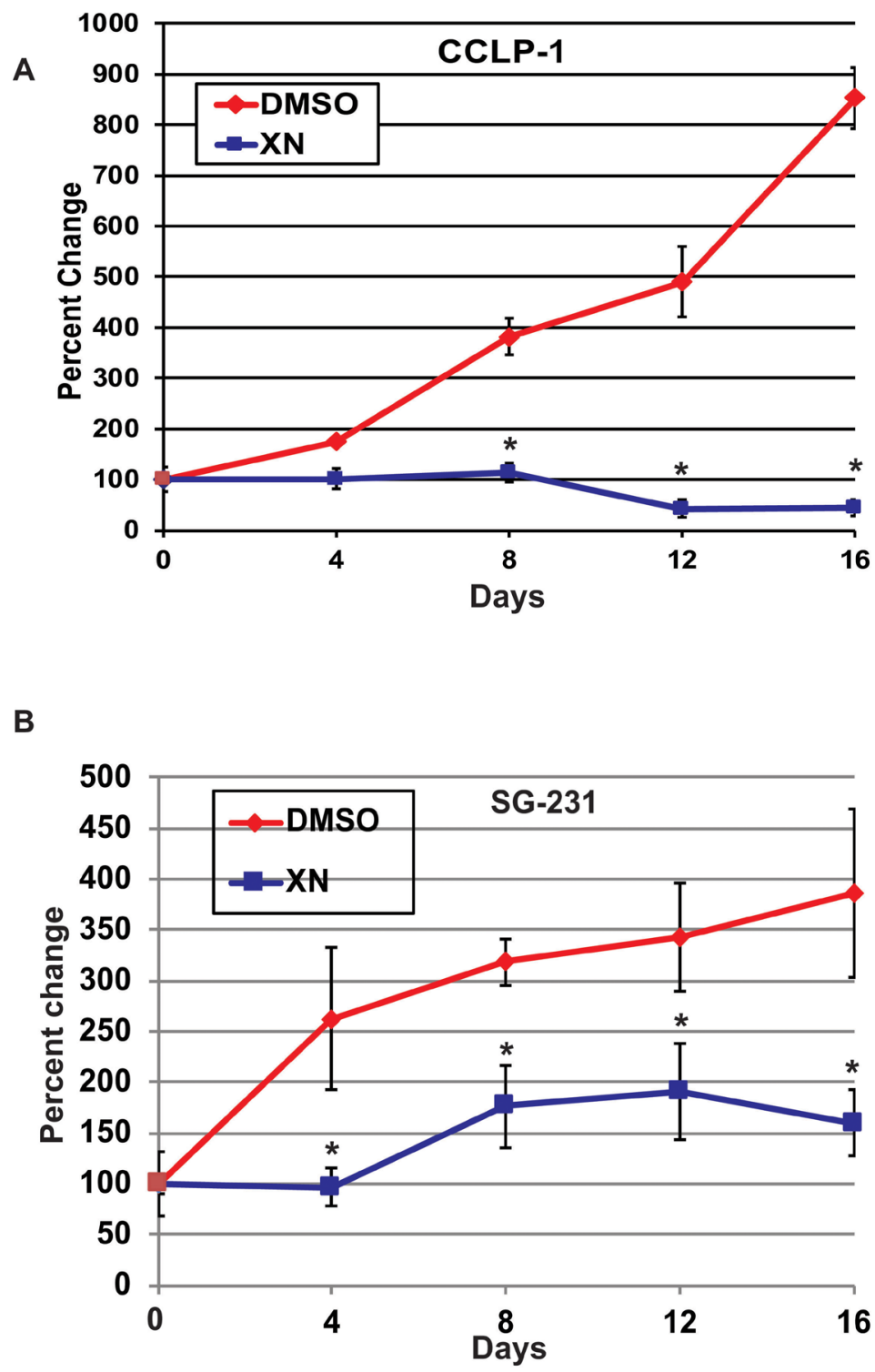

Figure 4: XN treatment inhibits tumor growth in both CCLP-1 and SG-231 cells injected in a mice xenograft model. (A) CCLP cells 8 days after treatment control mice demonstrated $381 \%$ change in tumor volume compared to a XN treated mice which showed a $13 \%$ decrease in tumor volume. After 16 days, control mice had a tumor volume $853 \%$ larger than on day 0 , compared to XN treated mice, which had a 78\% reduction in tumor volume. (B) SG-231 cells 8 days after injection untreated mice showed a $427 \%$-fold increase in tumor size compared to a $153 \%$ change in XN treated mice. After 16 days, control and XN treated mice demonstrated $1308 \%$ and $255 \%$-fold changes, respectively. CCLP-1 developed tumor did not increase in percentage fold after XN treatment throughout the treatment time points where as in SG-231 tumors there was increase in tumor fold and maintained the level during treatment compared to control treatment. Data were represented as $+/$ - s.e.m. ${ }^{*}, \mathrm{p}<0.001$. 
rate [20]. Thus, the need for advanced treatment, early diagnosis, and cellular understanding of CCA is critical.

Notch1 signaling is associated with proliferation, chemoresistance, metastasis, tumorigenesis and is overexpressed in many organ-specific cancers including liver and cholangiocarcinoma [21, 22]. Additionally, Notch1 is an important regulator of cellular proliferation in cancer stem cells, a small subset of cancer cells known to promote the tumor microenvironment and drive tumorigenesis [23]. Recently, Notch1 inhibition has shown to deplete the cancer stem cell population in gastric, breast, and glioma cancers through inhibiting angiogenesis and sensitizing the tumors to chemotherapy [23-25]. Clinical trials targeting Notch are currently ongoing. A new $\gamma$-secretase inhibitor, MK-0752, is currently in a phase I clinical trial for patients with pancreatic cancer. Primary results show there is a therapeutic benefit when given with gemcitabine in 11 out of 18 patients exhibiting progression free disease and confirmed partial response (NCT01098344). Additionally, a novel histone deacetylase with anti-Notch1 characteristics is currently in a phase I clinical trial as a potential therapy for medullary thyroid cancer (NCT01013597).

In this study, our results have confirmed that xanthohumol reduces Notch1 expression leading to apoptosis. Our data suggests that XN inhibits proliferation of CCA in both a dose and time dependent manner. The reduction of Notch1 levels after treatment in CCA is consistent with our previous data on hepatocellular carcinoma and pancreatic cancer [18, 19]. Therefore, we believe that Notch1 reduction is important for growth suppression by XN in CCA. Furthermore, Notch inhibition elicited the reduction in phosphorylation of AKT at the serine 473 residue without changing the levels of total AKT. AKT is overexpressed in roughly $70 \%$ of CCA cases and is strongly implicated in promotion of carcinogenesis in many cancers $[26,27]$. Furthermore, inhibition of AKT by LY294002 significantly suppressed human-growth factor-stimulated invasion in CCA by stabilization of E-cadherin [28]. Recently, we have shown that inhibition of the AKT pathway by MK-2206, AKT inhibitor, resulted in growth suppression [29].Therefore, we speculate that inhibition of both Notch and AKT pathways should be synergistic and more efficacious than inhibition of either pathway alone.

Our data provides evidence that $\mathrm{XN}$ inhibits human CCA (CCLP-1, SG-231, CC-SW-1) proliferation and induced apoptosis in both in vitro and in vivo models. We also demonstrated that XN inhibits Notch1 signaling as early as $12 \mathrm{hrs}$ after treatment. Reduction in phosphorylation of AKT at 3 days suggested that Notch signaling precedes and may initiate cross-talk with the PI3K/AKT signaling pathway. Furthermore, in vivo mice studies demonstrated inhibition of tumor progression with XN treatment.
Recent evidence into xanthohumol anti-tumorigenic abilities has been documented in various organ-specific cancer cell lines. Pharmacological studies suggest a large therapeutic window for XN and given a lower toxicity profile at lower concentrations, potential adverse effects are mitigated [30]. Such evidence suggests that XN could potentially be a novel agent for CCA. Our data warrants continued research into the efficacy of $\mathrm{XN}$ as a treatment strategy or adjunct therapy for CCA.

\section{MATERIALS AND METHODS}

\section{Cell Culture and XN Treatment}

Human CCA cell lines (CCLP-1 and SG-231), derived from intrahepatic biliary epithelium, were obtained from Dr. Anthony J. Demetris from the University of Pittsburgh. The CC-SW-1 cell line was kindly provided by Dr. Nabeel Bardeesy, Harvard Medical School. All cell lines were authenticated before receiving them. CCLP-1 cells were grown in Dulbecco Modified Eagle Medium (DMEM; Sigma-Aldrich, St. Louis, MO) with $1 \%$ penicillin/streptomycin (P/S; Life Technologies, Carlsbad, CA), 10\% fetal bovine serum (FBS; Life Technologies), $1 \%$ 4-(2-hydroxyethyl)-1-piperazineethanesulfonic (HEPES; Life Technologies), and 1\% non-essential amino acids (NEAA; Life Technologies). SG-231 cells were grown in Minimal Essential Medium alpha-1 (MEM $\alpha-$ 1; Life Technologies) with $10 \% \mathrm{FBS}, 1 \% \mathrm{P} / \mathrm{S}$, and $1 \%$ HEPES [16]. CC-SW-1 cells were grown in Roswell Park Memorial Institute (RPMI) 1640 medium with 10\% FBS and $1 \% \mathrm{P} / \mathrm{S}$. Cells were grown in a $37^{\circ} \mathrm{C}$ humidified incubator in $5 \% \mathrm{CO}_{2}$. Xanthohumol (Tocris, Minneapolis, MN or Selleckchem.com, Houston, TX) was dissolved in dimethyl sulfoxide (DMSO; Sigma-Aldrich).

\section{Cellular viability assay}

CCLP-1, SG-231, and CC-SW-1 cellular viability was measured through 3-(4, 5-dimethylthiazol-2-yl)-2, 5-diphenyltetrazolium bromide colorimetric assay (MTT). Cells were plated onto 96-well plates and allowed to adhere overnight. XN was added in 5, 10, and $15 \mu \mathrm{M}$ concentrations in triplicate. The control wells were treated with DMSO. Cells were incubated for either 48 or 96 hours. Media was replaced with $50 \mu \mathrm{L}$ RPMI1640 containing $0.5 \mathrm{mg} / \mathrm{mL}$ MTT and incubated for 3 hours. Following incubation, $150 \mu \mathrm{L}$ of DMSO was added to each well. Cellular absorbance was measured at $540 \mathrm{~nm}$ (Infinite M200 Pro; Tecan, San Jose, CA). Absorbance values were then calculated as percent cellular viability by dividing absorbance of the respective treatments groups by the control group. Statistical analysis was performed using the online software GraphPad ( $\mathrm{La}$ Jolla, CA). Unpaired t-tests were performed on each data set and P-values $<0.05$ were considered significant. 


\section{Colony formation assay}

To further assess proliferation and efficacy of $\mathrm{XN}$, colony formation assay was performed on these three cell lines. Cells were plated onto 6-well plates and allowed to adhere overnight. Cells were treated with DMSO (control), 5,10 , or $15 \mu \mathrm{M}$ concentrations of $\mathrm{XN}$ for three days and then media was changed every 3 days without XN for 2 weeks. Cells were then fixed with crystal violet and images were obtained with Molecular Imager Chemi-Doc XRS ${ }^{+}$imager with Image Lab software (Bio-Rad, Hercules, CA).

\section{Non-invasive cellular proliferation assay in real time}

Using IncuCyte Live-Cell imaging system (Essen Bioscience), cellular proliferation of CCA cell lines were measured as described [19]. Cell confluence was calculated using IncuCyte 2011A software and the cell proliferation was expressed as an increase in percentage of confluence with different time intervals.

\section{Western blot analysis}

Cell lysates were obtained following 3 days of $\mathrm{XN}$-treatment using the radioimmunoprecipitation assay buffer (RIPA; Thermo Fisher Scientific. Waltham, Mass). Protein quantification was measured by BCA protein assay (Thermo Scientific). Thirty micrograms of protein were loaded on to $7.5 \%, 10 \%$, or $12 \%$ sodium dodecyl sulfate polyacrylamide gels (Bio-Rad Laboratories). Gels were run until the proteins separated and were transferred onto a nitrocellulose membrane using the Trans-Blot Turbo Transfer System (Bio-Rad). Transferred membranes were blocked in a $5 \%$ dry milk solution and incubated in primary antibody overnight at $4^{\circ} \mathrm{C}$. Primary antibodies used were: Notch1 (1:1000), Survivin (1:500), Cyclin D1 (1:500), total and phosphorylated AKT (1:1000), p21 (1:200), total poly-ADP ribose polymerase (PARP) (1:2000) and glyceraldehyde 3-phosphate dehydrogenase (GAPDH) (1:4000) all from Santa Cruz Biotechnologies, Dallas, TX), cleaved PARP, X-linked inhibitor of apoptosis protein (XIAP), Cyclin D3, Cyclin E1, CDK2, and CDK4 all (1:1000) from Cell signaling Technology, Boston, MA. After overnight incubation, membranes were washed three times in a $1 \mathrm{x}$ phosphate buffered solution with $0.05 \%$ Tween-20 buffer. Following membrane wash, the blots were incubated with horse-radish peroxidase linked antimouse or anti-rabbit secondary antibody (1: 5,000, Santa Cruz) depending on the source of the primary antibody. The membranes were developed using Luminol (Santa Cruz) Supersignal West Dura or West Femto (Thermo Fisher Scientific). The membranes were then imaged using the Molecular Images Chemi-Doc $\mathrm{XRS}^{+}$imager (Bio-Rad).

\section{Caspase- 3 and -7 activities}

Caspase-Glo 3/7 Assay (Promega, Madison, WI) kit was used to measure the active caspase 3 and -7 from the lysates of cells treated with XN as described [19]. Luminescence was measured using Infinite M200PRO Microplate reader (TECAN).

\section{Cholangiocarcinoma tumor xenografts in mice}

CCLP-1 or SG-231 ( 1 X $10^{6}$ cells in $100 \mu$ l) were subcutaneously injected into the flank of 6-week-old $\mathrm{BALB} / \mathrm{c}$ nude mice obtained from the Charles laboratory. Treatments (125 ug of XN dissolved in saline was injected intraperitoneally into the treatment group every other day) were started after palpable tumors were seen. The mice were divided into two groups $(\mathrm{n}=5)$ and treated with either control (saline) or XN in saline for every other day for a total of 16 days. Mice tumors were measured every 4 days and the tumor volume was calculated using the formula $\mathrm{X}^{2} \mathrm{Y} / 2$, where $\mathrm{x}$ is the smallest and $\mathrm{y}$ is the largest diameters. Percentage of fold change was calculated.

\section{ACKNOWLEDGMENTS}

Thanks to Victoriana Schwartz for reviewing this manuscript. We appreciate the reviewers for their helpful comments on this article.

\section{CONFLICTS OF INTEREST}

Authors declare no conflicts of interest.

\section{GRANT SUPPORT}

The current study was supported in part by The Medical College of Wisconsin's Dr. Michael J. Dunn Summer Research Fellowship (DW), the Jack H Stein Memorial Research Program, and Dean's development program. Contributions of the authors are as follows: Walden D, Sokolowski K, Kunnimalaiyaan S, Gamblin $\mathrm{TC}$, Kunnimalaiyaan $\mathrm{M}$ aided in the concept and design of the project. Walden D and Kunnimalaiyaan S, Sokolowski K contributed to manuscript preparation and data collection. Gamblin TC and Kunnimalaiyaan M interpreted the results, analysis of the data, and revision of the manuscript.

\section{REFERENCES}

1. Chung V. Systemic therapy for hepatocellular carcinoma and cholangiocarcinoma. Surg Oncol Clin N Am. 2015; 24: 187-98. https://doi.org/10.1016/j.soc.2014.09.009.

2. Farley DR, Weaver AL, Nagorney DM. "Natural history" of unresected cholangiocarcinoma: patient outcome after 
noncurative intervention. Mayo Clin Proc. 1995; 70: 425-9. https://doi.org/10.1016/S0025-6196(11)63877-9.

3. Blechacz BR, Gores GJ. Cholangiocarcinoma. Clin Liver Dis. 2008; 12: 131-50, ix. https://doi.org/10.1016/j. cld.2007.11.003.

4. Shaib Y, El-Serag HB. The epidemiology of cholangiocarcinoma. Semin Liver Dis. 2004; 24: 115-25. https://doi.org/10.1055/s-2004-828889.

5. Rizvi S, Borad MJ, Patel T, Gores GJ. Cholangiocarcinoma: molecular pathways and therapeutic opportunities. Semin Liver Dis. 2014; 34: 456-64. https://doi. org/10.1055/s-0034-1394144.

6. Haigh WG, Lee SP. Identification of oxysterols in human bile and pigment gallstones. Gastroenterology. 2001; 121: 118-23.

7. Sekiya S, Suzuki A. Intrahepatic cholangiocarcinoma can arise from Notch-mediated conversion of hepatocytes. J Clin Invest. 2012; 122: 3914-8. https://doi.org/10.1172/ JCI63065.

8. Artavanis-Tsakonas S, Rand MD, Lake RJ. Notch signaling: cell fate control and signal integration in development. Science. 1999; 284: 770-6.

9. Lai EC. Notch signaling: control of cell communication and cell fate. Development. 2004; 131: 965-73. https://doi. org/10.1242/dev.01074.

10. Ai X, Jia Z, Liu S, Wang J, Zhang X. Notch-1 regulates proliferation and differentiation of human bladder cancer cell lines by inhibiting expression of Kruppel-like factor 4. Oncol Rep. 2014; 32: 1459-64. https://doi.org/10.3892/ or.2014.3350.

11. Xiao W, Chen X, He M. Inhibition of the Jagged/Notch pathway inhibits retinoblastoma cell proliferation via suppressing the PI3K/Akt, Src, p38MAPK and Wnt/ betacatenin signaling pathways. Mol Med Rep. 2014; 10: 453-8. https://doi.org/10.3892/mmr.2014.2213.

12. Kim SJ, Lee HW, Baek JH, Cho YH, Kang HG, Jeong JS, Song J, Park HS, Chun KH. Activation of nuclear PTEN by inhibition of Notch signaling induces $\mathrm{G} 2 / \mathrm{M}$ cell cycle arrest in gastric cancer. Oncogene. 2015. https://doi.org/10.1038/ onc.2015.80.

13. Fan B, Malato Y, Calvisi DF, Naqvi S, Razumilava N, Ribback S, Gores GJ, Dombrowski F, Evert M, Chen X, Willenbring $\mathrm{H}$. Cholangiocarcinomas can originate from hepatocytes in mice. J Clin Invest. 2012; 122: 2911-5. https://doi.org/10.1172/JCI63212.

14. Moeini A, Sia D, Bardeesy N, Mazzaferro V, Llovet JM. Molecular Pathogenesis and Targeted Therapies for Intrahepatic Cholangiocarcinoma. Clin Cancer Res. 2016; 22: 291-300. https://doi.org/10.1158/1078-0432. CCR-14-3296.

15. Zhou Q, Wang Y, Peng B, Liang L, Li J. The roles of Notch1 expression in the migration of intrahepatic cholangiocarcinoma. BMC Cancer. 2013; 13: 244. https:// doi.org/10.1186/1471-2407-13-244.
16. Koprowski S, Sokolowski K, Kunnimalaiyaan S, Gamblin TC, Kunnimalaiyaan M. Curcumin-mediated regulation of Notch1/hairy and enhancer of split-1/survivin: molecular targeting in cholangiocarcinoma. J Surg Res. 2015; 198: 434-40. https://doi.org/10.1016/j.jss.2015.03.029.

17. Jongthawin J, Techasen A, Loilome W, Yongvanit P, Namwat N. Anti-inflammatory agents suppress the prostaglandin E2 production and migration ability of cholangiocarcinoma cell lines. Asian Pac J Cancer Prev. 2012; 13 Suppl: 47-51.

18. Kunnimalaiyaan S, Sokolowski KM, Balamurugan M, Gamblin TC, Kunnimalaiyaan M. Xanthohumol inhibits Notch signaling and induces apoptosis in hepatocellular carcinoma. PLoS One. 2015; 10: e0127464. https://doi. org/10.1371/journal.pone.0127464.

19. Kunnimalaiyaan S, Trevino J, Tsai S, Gamblin TC, Kunnimalaiyaan M. Xanthohumol-Mediated Suppression of Notch1 Signaling Is Associated with Antitumor Activity in Human Pancreatic Cancer Cells. Mol Cancer Ther. 2015; 14: 1395-403. https://doi.org/10.1158/1535-7163. MCT-14-0915.

20. DeOliveira ML, Cunningham SC, Cameron JL, Kamangar F, Winter JM, Lillemoe KD, Choti MA, Yeo CJ, Schulick RD. Cholangiocarcinoma: thirty-one-year experience with 564 patients at a single institution. Ann Surg. 2007; 245: 755-62. https://doi.org/10.1097/01. sla.0000251366.62632.d3.

21. Zhou L, Wang DS, Li QJ, Sun W, Zhang Y, Dou KF. The down-regulation of Notch1 inhibits the invasion and migration of hepatocellular carcinoma cells by inactivating the cyclooxygenase-2/Snail/E-cadherin pathway in vitro. Dig Dis Sci. 2013; 58: 1016-25. https://doi.org/10.1007/ s10620-012-2434-7.

22. Zhou L, Zhang N, Song W, You N, Li Q, Sun W, Zhang Y, Wang D, Dou K. The significance of Notch1 compared with Notch3 in high metastasis and poor overall survival in hepatocellular carcinoma. PLoS One. 2013; 8: e57382. https://doi.org/10.1371/journal.pone.0057382.

23. Yan B, Liu L, Zhao Y, Xiu LJ, Sun DZ, Liu X, Lu Y, Shi J, Zhang YC, Li YJ, Wang XW, Zhou YQ, Feng SH, et al. Xiaotan Sanjie decoction attenuates tumor angiogenesis by manipulating Notch-1-regulated proliferation of gastric cancer stem-like cells. World J Gastroenterol. 2014; 20 : 13105-18. https://doi.org/10.3748/wjg.v20.i36.13105.

24. Qiu M, Peng Q, Jiang I, Carroll C, Han G, Rymer I, Lippincott J, Zachwieja J, Gajiwala K, Kraynov E, Thibault $\mathrm{S}$, Stone D, Gao Y, et al. Specific inhibition of Notch1 signaling enhances the antitumor efficacy of chemotherapy in triple negative breast cancer through reduction of cancer stem cells. Cancer Lett. 2013; 328: 261-70. https://doi. org/10.1016/j.canlet.2012.09.023.

25. Wang J, Wang C, Meng Q, Li S, Sun X, Bo Y, Yao W. siRNA targeting Notch-1 decreases glioma stem cell proliferation and tumor growth. Mol Biol Rep. 2012; 39: 2497-503. https://doi.org/10.1007/s11033-011-1001-1. 
26. Chung JY, Hong SM, Choi BY, Cho H, Yu E, Hewitt SM. The expression of phospho-AKT, phospho-mTOR, and PTEN in extrahepatic cholangiocarcinoma. Clin Cancer Res. 2009; 15: 660-7. https://doi.org/10.1158/1078-0432. CCR-08-1084.

27. Schmitz KJ, Lang H, Wohlschlaeger J, Sotiropoulos GC, Reis H, Schmid KW, Baba HA. AKT and ERK1/2 signaling in intrahepatic cholangiocarcinoma. World J Gastroenterol. 2007; 13: 6470-7.

28. Menakongka A, Suthiphongchai T. Involvement of PI3K and ERK1/2 pathways in hepatocyte growth factor-induced cholangiocarcinoma cell invasion. World J Gastroenterol. 2010; 16: 713-22.
29. Wilson JM, Kunnimalaiyaan S, Gamblin TC, Kunnimalaiyaan M. MK2206 inhibits hepatocellular carcinoma cellular proliferation via induction of apoptosis and cell cycle arrest. J Surg Res. 2014; 191: 280-5. https:// doi.org/10.1016/j.jss.2014.05.083.

30. Jiang W, Zhao S, Xu L, Lu Y, Lu Z, Chen C, Ni J, Wan $\mathrm{R}$, Yang L. The inhibitory effects of xanthohumol, a prenylated chalcone derived from hops, on cell growth and tumorigenesis in human pancreatic cancer. Biomed Pharmacother. 2015; 73: 40-7. https://doi.org/10.1016/j. biopha.2015.05.020. 